\title{
Edelfalter im Palmengarten und Botanischen Garten
}

\author{
Alfred Westenberger, Marco Schmidt und Hilke Steinecke
}

\begin{abstract}
In 2018 the Palmengarten started on iNaturalist the citizen science project "Tiere und Pilze in Frankfurts Botanischen Gärten". This article describes the twelve observed species of brush-footed butterflies (Nymphalidae), the family with the most colourful of our indigenous butterfly species.
\end{abstract}

\section{Zusammenfassung}

Im Jahr 2018 initiierte der Palmengarten das Bürgerwissenschaftsprojekt „Tiere und Pilze in Frankfurts Botanischen Gärten“. Dieser Beitrag beschreibt die zwölf beobachteten Arten der Edelfalter (Nymphalidae), der Familie mit den farbenprächtigsten Arten unserer heimischen Schmetterlinge.

\section{Einleitung}

Die Edelfalter (Nymphalidae) sind weltweit mit etwa 6000 Arten verbreitet und bilden die vielfältigste Familie unter den Tagfaltern. Man kann diese mittelgroßen bis großen, überwiegend farbenprächtigen Schmetterlinge, praktisch auf allen Erdteilen antreffen. Die meisten Arten der Familie kommen in den südamerikanischen Tropen vor. Europa beheimatet knapp 250 dieser Falterarten und in Deutschland allein kommen, mit Ausnahme der alpinen Arten an unserer südlichen Landesgrenze (z.B. die vielen Mohrenfalter), etwa 65 Edelfalterarten vor. Frankfurt am Main, mit der näheren Umgebung und dem Taunus einbezogen, beherbergt aktuell 28 dieser schönen Tagfalterarten.

Ein Großteil der Edelfalterarten imponiert durch sehr bunte Flügeloberseiten, wie beispielsweise der Kleine Fuchs und das Tagpfauenauge. Die Unterseiten der Flügel sind bei diesen Arten oftmals nur einfarbig oder unauffällig gefärbt. Edelfalter erreichen eine Flügelspannweite von etwa 4 bis $10 \mathrm{~cm}$, haben aber im Gegensatz zu anderen Tagfalterfamilien ein markantes Unterscheidungsmerkmal: Ihr erstes Beinpaar ist zu Putzpfoten verkümmert (daher der englische Name „brush-footed butterflies“!) und in Ruhestellung oder bei der Nahrungsaufnahme sitzen sie nur auf vier Beinen.

Bei sonnigem Wetter und Temperaturen über $20^{\circ} \mathrm{C}$ können einige der heimischen Edelfalterarten auch regelmäßig im Palmengarten und im
Botanischen Garten angetroffen werden. Die in unseren Gärten vorhandenen Kleinlebensräume, mit blumenreichen Wiesen, Waldbäumen, Sträuchern und Gebüschen sorgen dafür, dass sich sowohl einige Waldarten der Edelfalter, wie z.B. der Kaisermantel, der Kleine Schillerfalter, der Große Fuchs und das Waldbrettspiel, als auch verschiedene Wiesenfalterarten, wie beispielsweise der Schachbrettfalter, das Große Ochsenauge und das Kleine Wiesenvögelchen, wohlfühlen. Dazu gesellen sich auf den blühenden Wiesenteppichen unserer Gärten zur Nektaraufnahme auch unsere heimischen Brennnesselfalter, wie der Kleine Fuchs, das Tagpfauenauge und der C-Falter. Auch die als Wanderfalter bekannten Arten, der Distelfalter und der Admiral, können hier bis zum Spätsommer beobachtet werden. Mit gewissem Stolz können wir daher feststellen, dass seit Beginn unseres im Jahre 2018 auf der iNaturalist-Plattform ins Leben gerufenen Bürgerwissenschaftsprojekts in unseren Gärten insgesamt zwölf Edelfalterarten beobachtet und fotografisch festgehalten wurden. Um diese schönen Tagfalterarten näher kennenzulernen, stellen wir sie nachstehend in Wort und Bild vor.

\section{Kleiner Fuchs (Aglais urticae)}

Der Kleine Fuchs, auf den Flügeloberseiten mit einer rötlich-orangebraunen Grundfarbe, schwarzen Punkten, gelblichen und weißen Bereichen sowie mit leuchtend blauen, mondförmigen Randflecken versehen, ist einer unserer schönsten heimischen Tagfalter. Er ist mit keiner anderen Tagfalterart zu verwechseln und gehört zu den vier 
heimischen „Nesselfalterarten“, deren Raupen ausschließlich die Blätter der Großen Brennnessel als Nahrung aufnehmen.

Im Frankfurter Raum kommt der Kleine Fuchs in zwei bis drei Generationen vor. In unseren Botanischen Gärten kann man ihn in klimabegünstigt warmen Jahren sogar bis in den Oktober hinein bei der Nektaraufnahme an den letzten noch blühenden Pflanzen beobachten. Bei der Nahrungsaufnahme ist der Kleine Fuchs nicht wählerisch. Im Frühling kann man ihn in unseren Gärten z.B. an Huflattich, Löwenzahn, Seidelbast und Weidenkätzchen beobachten. Der nächsten Generation im Frühsommer stehen dann eine Vielzahl anderer Blütenpflanzen, wie beispielsweise die Wiesen-Flockenblume, Witwenblume, Skabiose, Gewöhnlicher Dost, Blaue Luzerne, Rotklee und die Acker-Kratzdistel als Nahrungspflanze zur Verfügung. Bei den Nektar spendenden Pflanzen darf keinesfalls der ab Juli bis in den Oktober blühende Schmetterlingsflieder vergessen werden. Wegen seiner außergewöhnlich guten Nektargabe wirken seine Blüten bekanntermaßen auf Schmetterlinge und auch andere Insekten, wie Bienen, Hummeln und Käfer, wie ein Magnet.

Die letzte Generation des Kleinen Fuchses überwintert als Falter. Hierzu suchen die Schmetter-

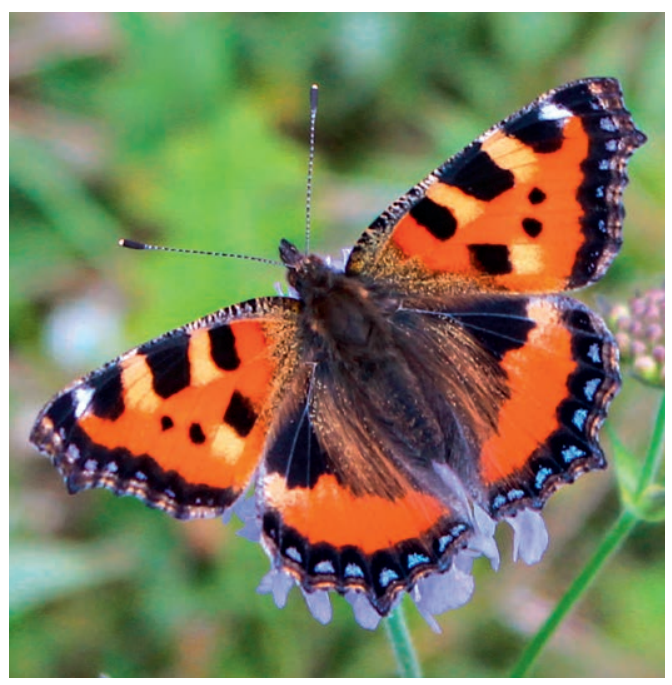

Abb. 1: Kleiner Fuchs auf einer Acker-Witwenblume. (Foto: A. Westenberger) linge geschützte Überwinterungsverstecke auf. Dachböden, Kellerräume, Gartenhäuser, Schuppen, Fels- und Erdhöhlen sind hier willkommene Plätze. Hier setzen sich die Überwinterer vorwiegend an den Decken fest, fahren ihren Stoffwechsel herunter, produzieren das körpereigene Frostschutzmittel Glycerin und fallen dann in eine Art Kältestarre. In diesem Zustand überwintern die Falter dann ca. 5 Monate und können so selbst $-20{ }^{\circ} \mathrm{C}$ schadlos überstehen.

Im Frühjahr, meistens an den ersten sonnigwarmen Frühlingstagen im April, beenden die Falter ihre Winterruhe. Ab dieser Zeit können sie dann bei der Suche nach den ersten Nektarpflanzen auch in unseren Gärten beobachtet werden. Wenn sich die Geschlechter im Frühling begegnen, kommt es bald zur Paarung. Dann dauert es nicht lange, bis die Weibchen ihre Eier, meist in Paketen von bis zu 200 Eiern, an der Blattunterseite der noch jungen Brennnesseltriebe ablegen. Etwa zehn Tage später schlüpfen die Räupchen. Diese leben zunächst gesellig in „Raupennestern“. Ab dem 3. Larvenstadium verteilen sich die Raupen auf dem Brennnesselbestand und leben dann nur noch einzeln an der Futterpflanze. Nach dem 4. Larvenstadium verpuppen sich die adulten Raupen und etwa acht bis zehn Tage später schlüpft der neu entwickelte Schmetterling der 1. Generation.

\section{Tagpfauenauge (Aglais io)}

Auch das Tagpfauenauge, das auf den Flügeloberseiten eine rotbraune Grundfärbung aufweist und mit schwarz-blau-rot-gelb gefärbten Augenflecken versehen ist, gehört zu unseren schönsten heimischen Edelfalterarten. Eine Verwechslung mit anderen heimischen Tagfalterarten ist auszuschließen. Mit zusammengefalteten Flügeln sieht der Falter schwärzlichbraun aus, erinnert an ein verwelktes Blatt und schützt sich so in Sträuchern und Gebüschen vor räuberischen Vögeln. Bei dieser Tarnungsform kann daher zu Recht von „Phytomimese“ gesprochen werden. Beobachtet hat man beim Tagpfauenauge auch, dass beim Öffnen der Flügel wegen der dann sichtbaren Augenflecke Vögel schnell wegfliegen, weil sie annehmen, ein angreifendes Tier vor sich zu haben. 


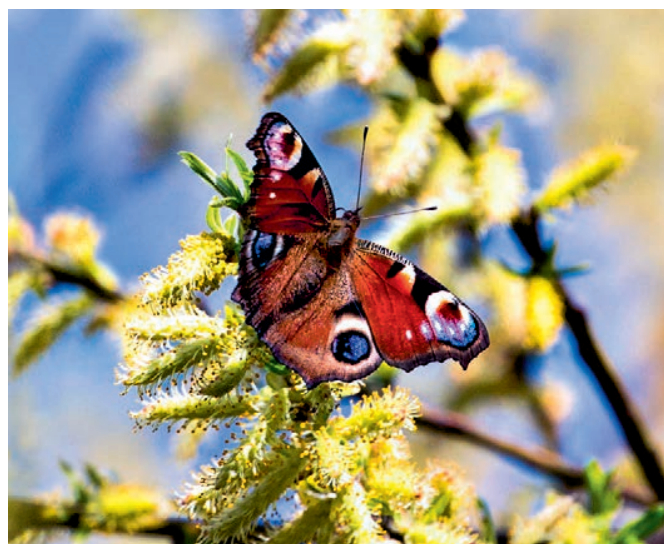

Abb. 2: Tagpfauenauge auf blühenden Weidenkätzchen. (Foto: G. HawicкhоRsт)

Das Tagpfauenauge gehört wie der Kleine Fuchs, der Zitronenfalter, der Faulbaumbläuling und der Aurorafalter zu unseren ersten Frühlingsboten. Es kann an vielen blumenreichen Stellen unserer Gärten beobachtet werden. Sowohl beim Blütenbesuch im Frühjahr und Sommer, als auch bei der im Herbst beginnenden Überwinterung hat es die gleiche Strategie wie der Kleine Fuchs. Selbst die Paarungszeit und die häufchenartige Eiablage (in Paketen bis zu 200 Eiern) an den Blattunterseiten von Brennnesseln ist identisch mit dem Verhalten des Kleinen Fuchses. Klare Unterschiede beider Arten gibt es nur im Aussehen der Schmetterlinge und bei den erwachsenen Raupen. Während die Grundfarbe der bedornten Raupen des Kleinen Fuchses schwärzlich und mit zwei gelben Rückenstreifen und je einem gelben Seitenstreifen versehen sind, weisen die ebenfalls bedornten Raupen des Tagpfauenauges eine tiefschwarze Farbe auf und sind zudem zahlreich mit kleinen weißen Punkten gesprenkelt. Das Tagpfauenauge erscheint in Raum Frankfurt in ein bis zwei Generationen.

\section{C-Falter (Polygonia c-album)}

Dieser attraktiv aussehende Edelfalter hat zwar nicht die Farbenpracht vom Kleinen Fuchs und Tagpfauenauge, fällt aber besonders durch seine auffällig gezackten Flügelränder auf. Aus diesem Grund ist er von anderen Tagfalterarten gut zu unterscheiden. Flügeloberseits ist der C-Falter orangebraun gefärbt, hat dunkle Flecken und ist mit einem dunkelbraunen Rand versehen. Die Unterseiten der Flügel sind graubraun gefärbt, rindenartig gezeichnet und auf den Hinterflügeln ist das namensgebende Merkmal, ein deutliches weißes „C“ zu sehen.

Die Lebensräume des C-Falters, der zweibrütig ist, sind lückige Wälder, Waldränder, -schneisen, -lichtungen und Gartenanlagen. Hier ist er oftmals bei der Nahrungsaufnahme an Disteln und Wasserdost zu beobachten. Er ist aber auch bei

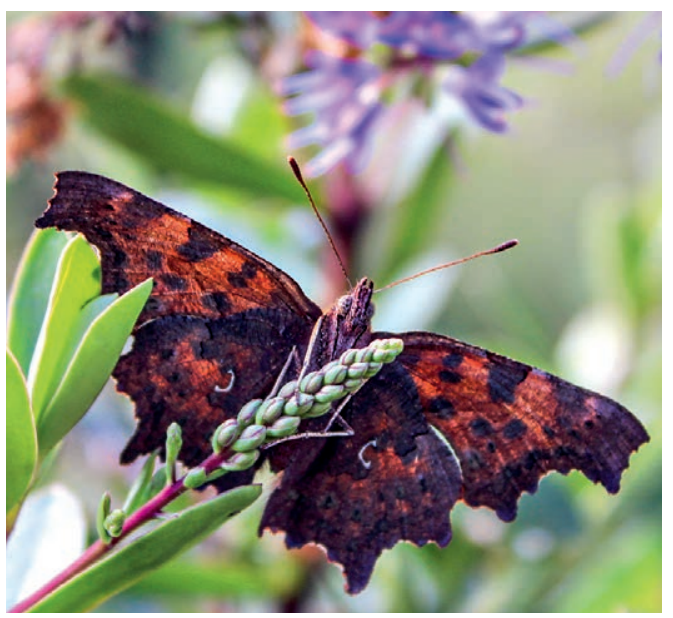

Abb. 3: Auf den Flügelunterseiten ist jeweils ein weißes $\mathrm{C}$ zu erkennen. Dunkle überwinternde Generation.

(Foto: A. Westenberger)

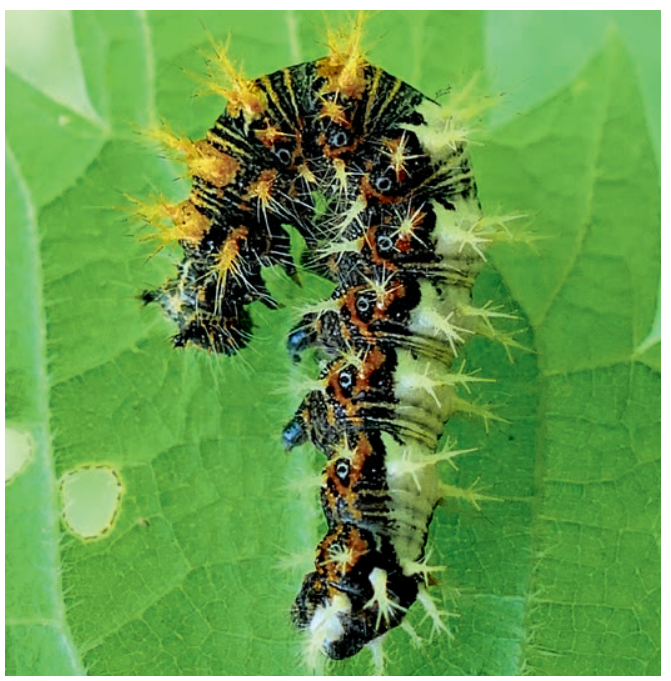

Abb. 4: Die Raupe des C-Falters imitiert Vogelkot und wird somit für Fressfeinde uninteressant. (Foto: H. Steinecke) 
der Mineralienaufnahme auf feuchten Waldwegen oder an tierischen Exkrementen saugend anzutreffen. Mit etwas Glück kann man in unseren Gärten im Frühjahr überwinterte Falter bei der Nektaraufnahme an Huflattich und Weidenkätzchen (Salweide) beobachten.

Außer der Großen Brennnessel nutzt der C-Falter u.a. Hasel, Ulme und Salweide als Eiablage- und Raupennahrungspflanze. Die imposante, bedornte Raupe, deren mittlerer und hinterer Teil des Rückens blütenweiß ist und damit Vogelkot nachahmt (sogenannte Vogelkot-Mimese) ist in Obstgärten sogar an Stachel- und Johannisbeere festgestellt worden. Der C-Falter überwintert als fertig entwickelter Falter. Als Überwinterungsplatz nimmt er allerdings keine menschlichen Behausungen an, sondern sucht hierzu Baumhöhlungen, aufgeschichtete Baumstämme, Erdhöhlen und dergleichen auf. Bei fast vollständig heruntergefahrenem Stoffwechsel übersteht so der Falter selbst extrem kalte Winter schadlos.

\section{Admiral (Vanessa atalanta)}

Auch der als Wanderfalter bekannte Admiral kann oft in unseren Gärten beobachtet werden. Er ist eine Augenweide unter den Edelfaltern und zählt zu den schönsten Tagfaltern Mitteleuropas. Die farbliche Kombination auf den Flügeloberseiten, mit der schwarzen Grundfärbung, den roten Binden und den Weißanteilen, sieht prächtig aus. Die roten Flügelbinden auf den Vorder- und Hinterflügeln sorgen u. a. dafür, dass der Admiral mit keiner unserer heimischen Tagfalterarten verwechselt werden kann. In früheren Jahren flog der Admiral bei Hochdruckwetter jährlich im Mai/Juni, aus dem mediterranen Süden kommend, bei uns ein. Mit der Klimaerwärmung und den milden Wintern der letzten Jahre gilt es mittlerweile als gesichert, dass der Falter in geschützten Lagen in Deutschland (z.B. der Freiburger Gegend, Kaiserstuhl; jetzt aber auch im Rhein-Main-Gebiet) überwintert. Das gibt ihm die Möglichkeit, jährlich drei Generationen hervorzubringen. Eiablageund Raupennahrungspflanze ist die Große Brennnessel, in deren Fruchtstände die Weibchen ihre Eier, jeweils aber nur einzeln (ein Ei pro Pflanze), ablegen. Der Admiral besiedelt die verschiedensten

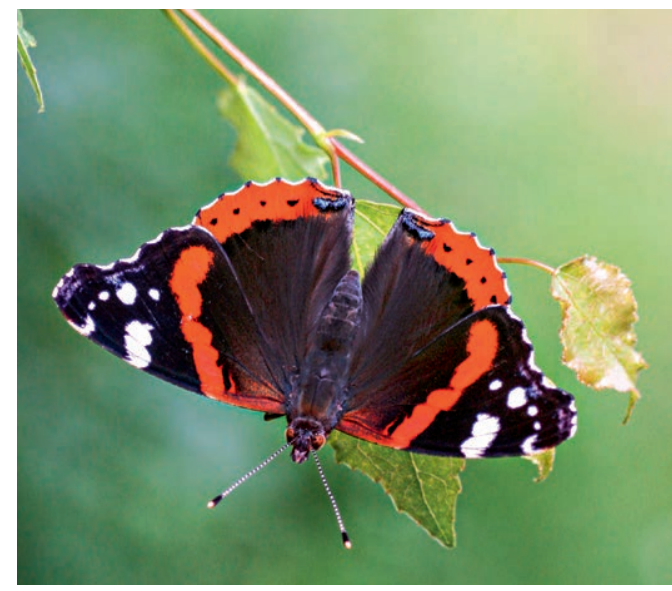

Abb. 5: Admiral auf einem Birkenzweig.

(Foto: A. Westenberger)

Lebensräume. Er ist sowohl in lichten Wäldern, an Waldwegen und -rändern, als auch in verschiedenen Offenlandbiotopen anzutreffen. Oft hält er sich auf Obstbaumgrundstücken und in Hausgärten auf, wobei für ihn der in Hausgärten vorhandene Schmetterlingsflieder eine willkommene Nahrungsquelle ist.

Im Spätsommer und Herbst sieht man die Falter bei sonnigem Wetter oft an überreifem Fallobst sitzen, um sich an den gärenden Säften zu laben. Wenn er sich zur Überwinterung nicht in südliche, wärmere Gefilde im Mittelmeerraum zurückzieht, überwintert er hier wie unsere heimischen Nesselfalterarten. Langanhaltender, starker Frost ist für den Admiral allerdings tödlich.

\section{Distelfalter (Vanessa cardui)}

Auch Deutschlands bekanntester Wanderfalter, der Distelfalter, kann im Sommer im Palmengarten und dem Botanischen Garten beobachtet werden. Erste Wellen dieser schön aussehenden Schmetterlinge fliegen bereits im Mai/ Juni aus dem südeuropäischen Mittelmeerraum und Nordafrika in unsere Gegend ein. Im Juli/ August folgen dann weitere Wellen von $\mathrm{Zu}$ wanderern. Da die Falter bei der Wanderung oft weite Strecken zurücklegen, sind sie an günstige Hochdruckwetterlagen gebunden. Die eingewanderten Distelfalter entwickeln bei uns zwei Nachfolgegenerationen. Ein Großteil der zweiten 


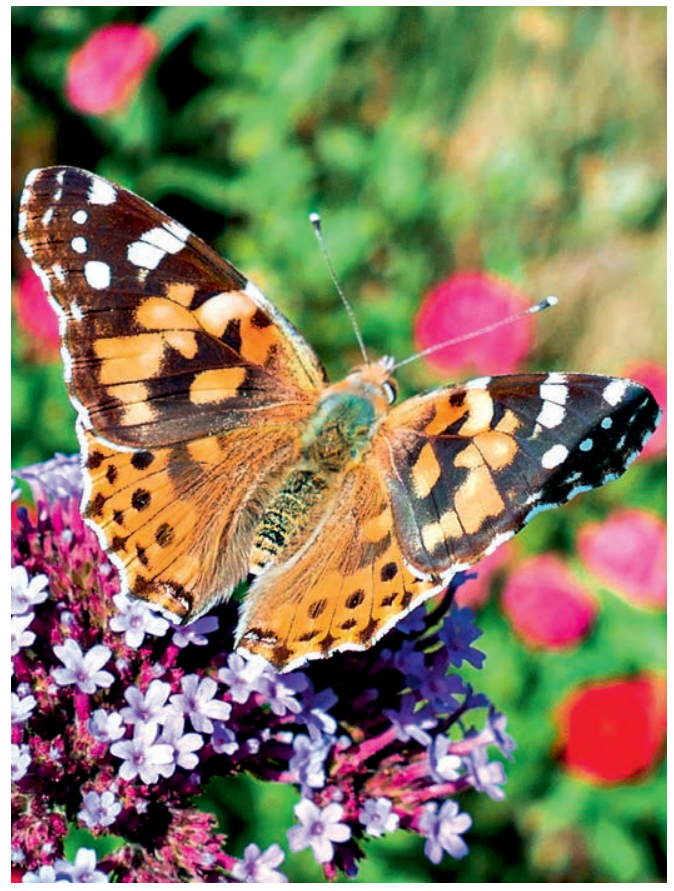

Abb. 6: Distelfalter an Patagonischem Eisenkraut.

(Foto: H. STEINECKe)

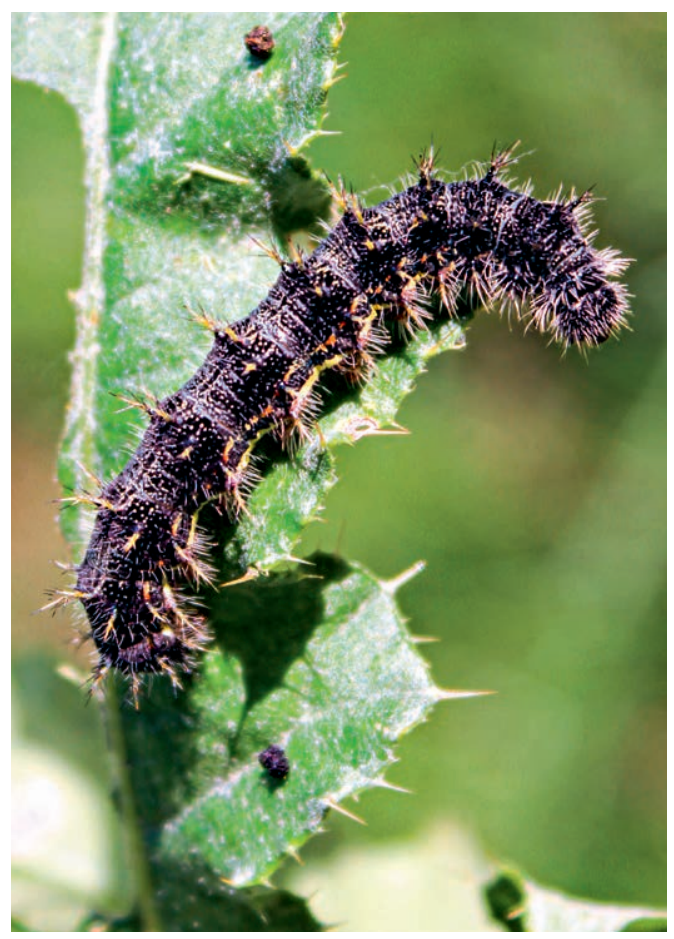

Abb. 7: Eine erwachsene Raupe des Distelfalters auf einer Gewöhnlichen Kratzdistel. (Foto: A. Westenberger)
Generation fliegt dann im Spätsommer wieder in den Süden zurück. Die hier verbliebenen Falter oder die, die den Rückzug nicht rechtzeitig vornehmen, überleben das kalte Herbst- und Winterwetter nicht. Fröste sind für die Distelfalter absolut tödlich.

Der Distelfalter ist eine Offenland-Art, der in blumenreichen Biotopen, wie Wiesen, Gärten und Parkanlagen, aber auch auf Ruderal- und Feldflächen mit Distelarten beobachtet werden kann. Neben verschiedenen Distelarten, vorzugsweise der Acker-Kratzdistel, wird auch die Große Brennnessel von den Weibchen zur Eiablage genutzt. Die Eier werden jeweils einzeln an den Blättern der Nahrungspflanze abgelegt. Die schwärzlich gefärbte, dornige Raupe ist mit ockergelben bis grünlich gelbbraunen Zeichnungen versehen. Man findet sie in einem lockeren Gespinst an der jeweiligen Futterpflanze.

\section{Großes Ochsenauge (Maniola jurtina)}

Das Große Ochsenauge ist in Mitteleuropa und auch im Frankfurter Raum einer der häufigsten Tagfalter. Obwohl er nicht die Farbenpracht anderer Edelfalterarten zeigt, kann man ihm eine gewisse Attraktivität trotzdem nicht absprechen. Die Männchen sind auf den Flügeloberseiten dunkelbraun gefärbt und weisen nahe der Vorderflügelspitzen einen schwärzlichen, weißgekernten Augenfleck auf. Die etwas größeren Weibchen haben im Gegensatz dazu auf den braunen Vorderflügeloberseiten eine deutliche, hellbraune Binde, auf der die Augenflecke zu sehen sind. Die Flügelunterseiten beider Geschlechter sind braun gefärbt, wobei die Weibchen zum Außenrand hin mit hellbeigen bis hellgrauen Binden gemustert sind. Sie wirken dadurch viel heller als die Männchen.

Lebensräume des einbrütigen Großen Ochsenauges sind blumenreiche Wiesen, Ruderalflächen, Luzerne- und Rotkleeäcker, Waldränder, Parkanlagen und Hausgärten. Man kann es von Juni bis September an den verschiedensten blühenden Blumen bei der Nektaraufnahme beobachten. Eine klare Präferenz liegt dabei für blaue und lilafarbene Blütenköpfchen vor. 


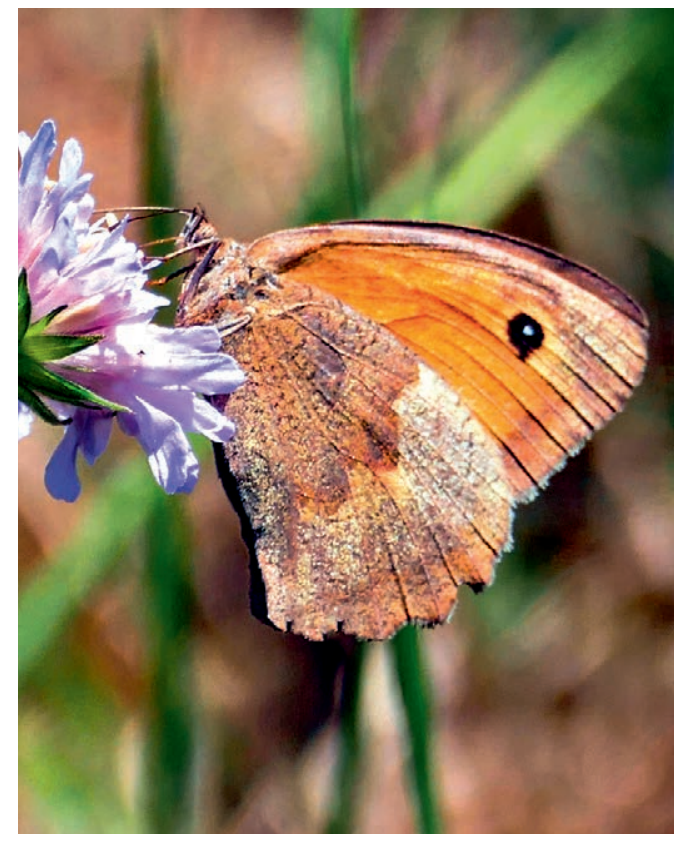

Abb. 8: Großes Ochsenauge an einer Acker-Witwenblume. (Foto: H. STeinecke)

Hauptsächlich die Weibchen des Großen Ochsenauges sind sehr langlebig. Sechs Wochen Lebenszeit sind keine Seltenheit. Zur Fortpflanzung legen die Weibchen ihre Eier an verschiedenen Grasarten, wie Rot-Schwingel, Fieder-Zwenke und besonders Wiesen-Rispengras ab. Beim Großen Ochsenauge überwintert die Raupe, und zwar im zweiten bis vierten Larvenstadium.

\section{Kleines Wiesenvögelchen \\ (Coenonympha pamphilus)}

Das Kleine Wiesenvögelchen, früher Kleiner Heufalter genannt, zählt zu den häufigsten Tagfaltern Deutschlands. Auch in Frankfurt ist der kleine Edelfalter (Flügelspannweite: 23 - $33 \mathrm{~mm}$ ) noch zahlreich anzutreffen. Mit seinen gelblichbraunen Flügeloberseiten, kaum sichtbaren dunklen Randbinden und durchleuchtenden Augenflecken an den Vorderflügelspitzen hat er ein recht unscheinbares Erscheinungsbild. In Ruhestellung und bei der Nektaraufnahme sieht man ihn fast nur mit zusammengefalteten Flügeln. Hierbei kann die graubraune, leicht gemusterte Hinterflügelunterseite gut erkannt werden. Beide Geschlechter se-

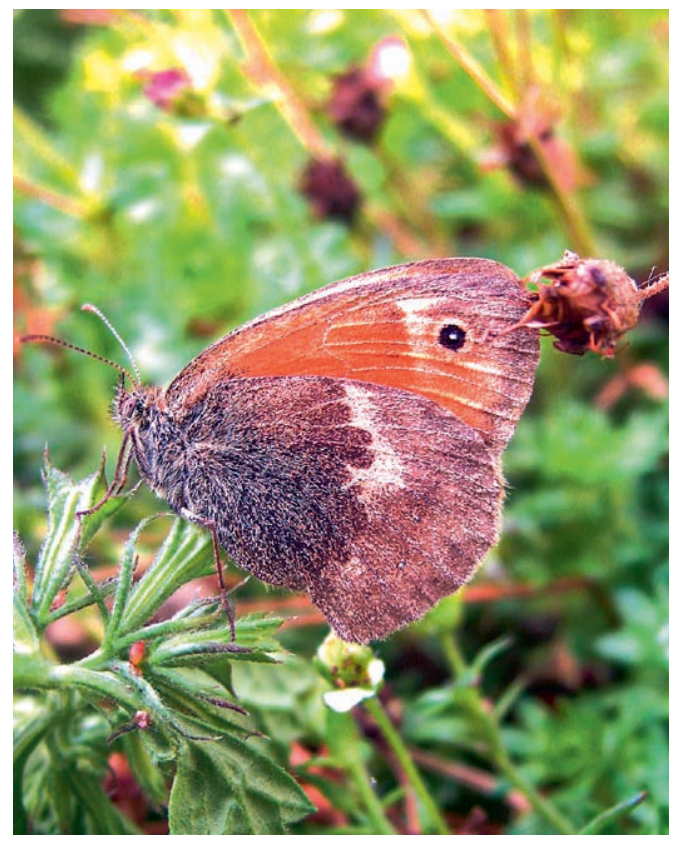

Abb. 9: Kleines Wiesenvögelchen.

(Foto: A. Westenberger)

hen nahezu gleich aus. Die Weibchen sind meistens etwas größer und fallen (wegen des Eivorrates) durch das dickere Abdomen auf. Verwechslungen mit anderen Tagfalterarten können in unserer Gegend ausgeschlossen werden.

Lebensräume des Kleinen Wiesenvögelchen sind offene, blumenreiche Graslandbiotope, wie z. B. ungedüngte Mähwiesen, Glatthaferwiesen, Straßen- und Waldränder sowie Parkanlagen. Im Frankfurter Raum tritt das Kleine Wiesenvögelchen in zwei bis drei Generationen auf. Auf den Wiesen in unseren Gärten kann es von Mai bis September, manchmal sogar bis in den Oktober hinein, beobachtet werden. Die kleinen Falter flattern dabei langsam und setzen sich oft an höherstehende Grashalme. Zur Nektaraufnahme werden z. B. Margerite, Hahnenfuß, Thymian, Schafgarbe, Weiß-Klee, Schneckenklee, Vogelknöterich, Wiesen-Flockenblume und auch der Schmetterlingsflieder aufgesucht. Zur Fortpflanzung legen die Weibchen ihre Eier an Gräsern wie Rot-Schwingel, Straußgrasarten und Wiesen-Rispengras ab. Die Jungraupe des Kleinen Wiesenvögelchens überwintert in der Bodenstreu. 


\section{Schachbrettfalter (Melanargia galathea)}

Der Schachbrettfalter mit seiner schwarz-weißen schachbrettartigen Flügelzeichnung ist unverwechselbar. Da die Weibchen des Schachbrettfalters meist etwas größer als die Männchen sind und deren Zeichnungen auf den Hinterflügelunterseiten ins ockerfarbene gehen, kann man die Geschlechter gut unterscheiden.

Im Frankfurter Raum ist der Schachbrettfalter noch recht gut vertreten. Ungedüngte blumige Wiesen, Brachflächen, Wald- und Wegsäume, Böschungen etc. sind seine zu schützenden Lebensräume. Der Schachbrettfalter fliegt jährlich in nur einer Generation. Ab etwa Mitte Juni bis in den August hinein kann er auf den blühenden Wiesen im Palmengarten und dem Botanischen Garten beobachtet werden. Bei der Nektaraufnahme bevorzugen die Falter blauviolette Blüten, wie z.B. Wiesen-Flockenblume, Witwenblume und Skabiosenarten.

Zur Fortpflanzung legen die Weibchen ihre Eier nicht an den Raupennahrungspflanzen, den Gräsern $\mathrm{ab}$, sondern sie lassen sie während des Flugs einfach in die Vegetation fallen (sogenannte Eierstreuer). Rot-Schwingel, Aufrechte Trespe und Pfeifengras sind einige der Gräser, die von den Raupen verzehrt werden.

Die Überwinterung erfolgt als Raupe. Das geschlüpfte Eiräupchen überwintert im ersten

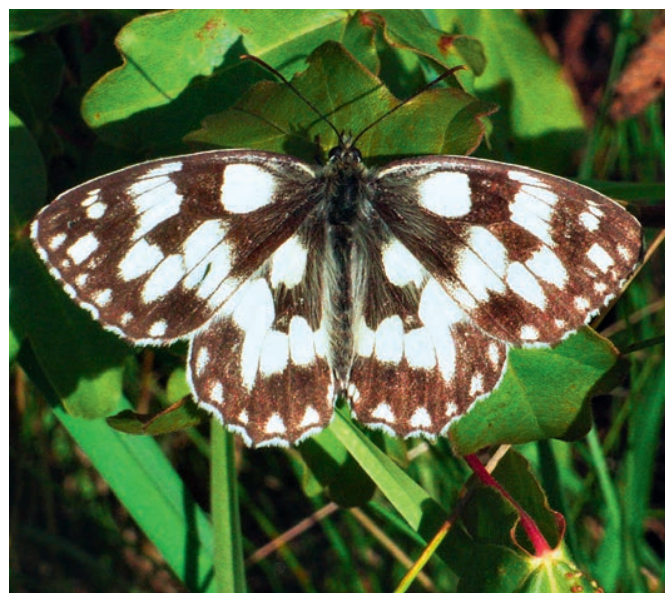

Abb. 10: Schachbrettfalter. (Foto: В. Јаковиs)
Larvenstadium. Wie an den „Augenflecken“ erkennbar, gehört er wie das Große Ochsenauge, das Kleine Wiesenvögelchen und das Waldbrettspiel der Unterfamilie Satyrinae, den Augenfaltern, an. Die erwähnten Augenfalterarten werden auch "Grasfalter" genannt, weil sich ihre Raupen ausnahmslos von Gräsern ernähren.

\section{Waldbrettspiel (Pararge aegeria)}

Auch das in den baumbestandenen Bereichen des Palmengartens und Botanischen Gartens anzutreffende Waldbrettspiel gehört zu den Edelfaltern. In ganz Mitteleuropa und auch in der Frankfurter Region ist es einer der häufigsten Augenfalter. Die Flügeloberseiten haben eine braune bis dunkelbraune Grundfarbe, die auf den Vorderflügeln mit mehreren weißen und hellbeigen Flecken und einem weiß gekernten Augenfleck versehen sind. Auf der Oberseite der Hinterflügel sind parallel zum Flügelaußenrand drei bis vier Augenflecke zu sehen, die teilweise auch weiß gekernt sind. Diese markanten Befleckungen sollten eine Verwechslung mit anderen heimischen Tagfalterarten ausschließen. Das Waldbrettspiel ist ein typischer Waldrandbewohner. Man trifft den Falter bei sonnigem Wetter im Licht-Schatten-Bereich grasiger Laubwälder und Waldwege, aber auch in Parkanlagen und Gärten an.

Die erste Generation des Waldbrettspiels fliegt schon im zeitigen Frühjahr. Mit etwas Glück können in unseren Gärten bereits ab Mitte April

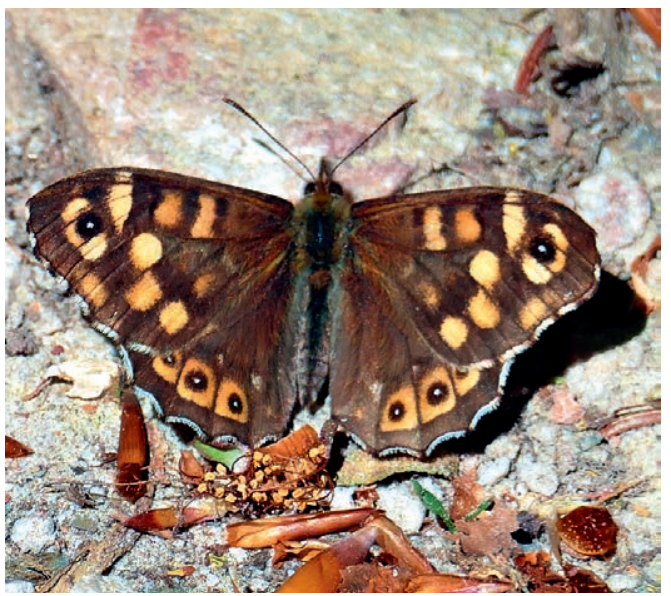

Abb. 11: Waldbrettspiel. (Foto: H. Steinecke) 
frisch geschlüpfte Falter beobachtet werden. Zur Nektaraufnahme saugt das Waldbrettspiel nur selten an Blüten. Häufiger sieht man den Falter bei der Mineralienaufnahme an feuchten Wegstellen. Auch beim Saugen an Wunden verschiedener Baumarten, reifen Früchten wie Himbeere und Brombeere und sogar an Fallobst wurde er beobachtet.

Bekannt ist das Revierverhalten der Männchen, die von einer erhöhten Sitzwarte aus andere in ihr Gebiet eindringende männliche Falter vertreiben. Vorbeifliegende Weibchen hingegen werden akzeptiert, wobei die Falter dann oftmals das umkreisende Liebesspiel beginnen. Zur Eiablage und damit zur Fortpflanzung nutzen die Weibchen etwa 20 verschiedene grasartige Arten. Einige wichtige davon sind z. B. Wald-Segge, Wald-Zwenke und Wiesen-Knäuelgras. In unserer Region ist das Waldbrettspiel in zwei bis drei Generationen zu sehen. Die Überwinterung erfolgt im Puppenstadium oder als halb erwachsene Raupe.

\section{Großer Fuchs (Nymphalis polychloros)}

Der Große Fuchs ähnelt in der Farbzusammensetzung und seiner Zeichnungen sehr dem Kleinen Fuchs. Vergleicht man die beiden Arten aber untereinander, kann man doch deutliche Abweichungen bei den Farben und Zeichnungen feststellen. Auch ist die Flügelspannweite (50-60 mm) beim Großen Fuchs größer (Kleiner Fuchs nur 40-50 mm). In unserer Region galt der Große Fuchs lange Zeit als verschollen. Die Gründe für das vorübergehende Verschwinden sind mannigfaltig. Unter anderem führt man hier die zeitweilige, übermäßige Anwendung von Pestiziden im Obstbau an, weil die Weibchen des Großen Fuchses zur Fortpflanzung auch Birne und Süßkirsche mit Eiern belegen.

Seit mittlerweile etwa zehn Jahren werden jedoch im Frühjahr sowohl im Frankfurter Raum als auch im Taunus-Gebiet wieder überwinterte, und im Sommer, zwischen Juni und August, Falter der ersten Generation festgestellt. Diese Beobachtungen sind äußerst positiv zu bewerten. Lebensräume des Großen Fuchses sind neben

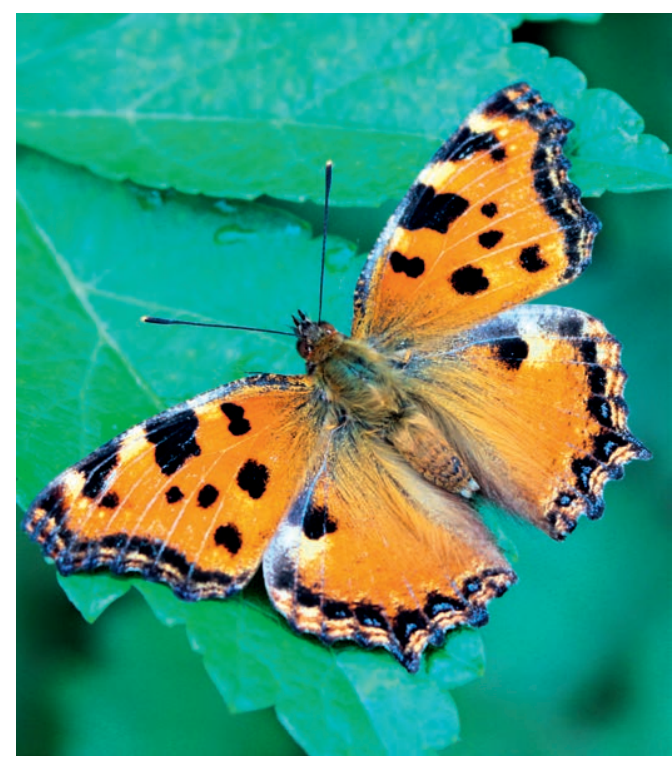

Abb. 12: Großer Fuchs. (Foto: A. Westenberger)

Obstbaumanlagen und Streuobstwiesen auch offene Wälder und sonnenexponierte Waldränder. Zur Eiablage bevorzugen die Weibchen in Waldbereichen Weidenarten (z.B. Salweide), Ulmen- und Pappelarten (z.B. Zitterpappel). Die Eier werden dabei in ringförmigen Gelegen um dünne Zweige abgelegt. Die geschlüpften Räupchen leben zunächst gesellig in einem lockeren Gespinst. Die größeren, dornenartig aussehenden Raupen vereinzeln sich später.

Die überwinternden Falter des Großen Fuchses sind mit die ersten Schmetterlinge, die man bei sonnigwarmem Wetter im Frühjahr beobachten kann. Bereits im März/April, zur Blütezeit von Schlehdorn, Kirsche und Zwetschge, kann sie der aufmerksame Naturfreund bei der Nektaraufnahme an den Blüten beobachten. Auch der im Frühling blühende Huflattich und blühende Weidenkätzchen werden als Nahrungsquelle gerne aufgesucht. An feuchten Stellen der Waldwege oder den Wegen von Parkanlagen kann der Falter oftmals bei der Mineralienaufnahme beobachtet werden. Hierzu werden von ihm auch auf Wegen liegende tierische Exkremente mit Vorliebe aufgesucht. Der einbrütige Große Fuchs überwintert als Falter in Baumhöhlen, aufgestapeltem Holz, Schuppen, Brücken und dergleichen. 


\section{Kaisermantel (Argynnis paphia)}

Der Kaisermantel ist der größte Perlmutterfalter Europas. Er ist ein typischer Vertreter der „Waldschmetterlinge" und ist in unseren heimischen Waldgebieten, z.B. auf Waldwegen, -schneisen, -lichtungen und Waldsäumen, erfreulicherweise noch häufig anzutreffen. Bei sonnigem Sommerwetter kann der imposante Edelfalter in den Monaten Juli und August beobachtet werden, wenn er entlang der Waldwege die weißen Blüten der Brombeere, die purpurroten des Wasserdostes oder die bläulichroten Blüten verschiedener Distelarten zur Nektaraufnahme aufsucht. Der Kaisermantel ist eine einbrütige Art. Wegen der silberfarbenen Längsbinden auf den Rückseiten seiner Hinterflügel wird er deshalb mancherorts auch „Silberstrich“ genannt und kann mit keiner anderen heimischen Tagfalterart verwechselt werden. Männchen und Weibchen des Kaisermantels sind auf den Flügeloberseiten unterschiedlich gefärbt und gezeichnet. Die Männchen des Kaisermantels weisen auf den Flügeloberseiten eine leuchtend orangebraune Farbe auf und haben auf den Vorderflügeln runde schwarze Flecken und kleinere Binden. Entlang der Flügeladern sind außerdem sehr deutlich vier längliche, schwarze Duftschuppenstriche zu sehen, dort werden Pheromone abgegeben. Die Weibchen haben dagegen

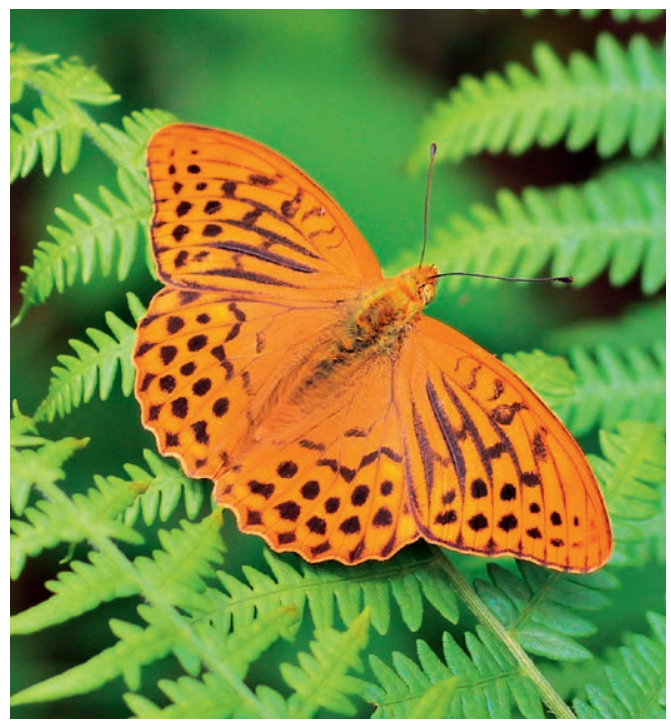

Abb. 13: Männchen des Kaisermantels mit „Duftschuppenstrichen". (Foto: A. Westenberger) flügeloberseits eine deutlich dunklere Grundfärbung und sind an den Adernbereichen, wo die Männchen mit Duftschuppenstrichen versehen sind, mit runden schwarzen Flecken gezeichnet. Erwähnenswert ist, dass bei den Weibchen des Kaisermantels neben der normalen Weibchenform auch die seltene, stark verdunkelte graue Weibchenform „valesina“ auftreten kann. Sie wird auch in unseren heimischen Wäldern immer wieder mal beobachtet. Raupennahrungspflanzen des Kaisermantels sind verschiedene Veilchenarten, wie beispielsweise das Wald-Veilchen und das März-Veilchen.

Die Eier werden nicht wie bei anderen Arten üblich, an den Fraßpflanzen der Raupen abgelegt, sondern an der Rinde von Baumstämmen in den mit Veilchen bewachsenen Saumbereichen. Dabei werden die Eier einzeln im unteren Bereich der Stämme in rissige Vertiefungen der Rindenteile abgelegt. Die im Herbst schlüpfenden Räupchen verkriechen sich anschließend in den Rindenritzen der Bäume und überwintern dort. Nach Beendigung der Winterruhe im Frühjahr begeben sich die winzigen Räupchen dann zur ersten Nahrungsaufnahme an die austreibenden Veilchen. Tagsüber leben die Raupen meist versteckt. In der Nacht gehen sie auf Nahrungssuche.

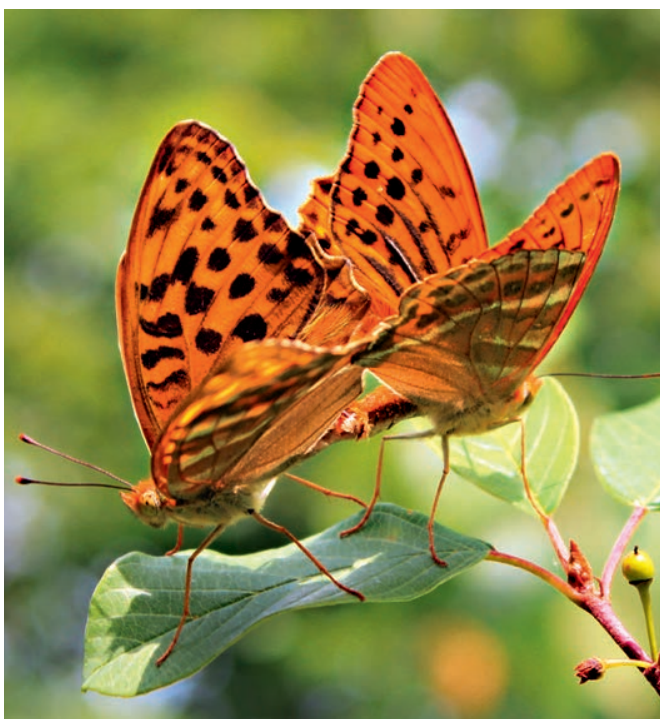

Abb. 14: Kopula des Kaisermantels auf einem Faulbaum, links das Weibchen. (Foto: A. Westenberger) 


\section{Kleiner Schillerfalter (Apatura ilia)}

Auch der Kleine Schillerfalter, hier insbesondere das blau schillernde Männchen der rötlichen Form clytie, ist ein herrlicher Tagfalter. Vom Aussehen steht er den prächtigsten Schmetterlingen tropischer Wälder nicht nach. Er hat eine Flügelspannweite von 64-72 mm. Auch er gehört zu unseren heimischen Waldarten. Bei sonnigem Wetter, hauptsächlich in den Monaten Juni und Juli, kann er auf den Wegen lichter Laub- oder Mischwälder mit Zitter- und Schwarzpappelbeständen beobachtet werden. Heiße und langanhaltende Sommer können begünstigen, dass sich beim Kleinen Schillerfalter eine partielle zweite Generation entwickelt. Diese Falter fliegen dann im August und September.

Interessant ist, dass er in Mitteleuropa und auch im Frankfurter Raum in zwei verschiedenen Farbmorphen vorkommt. Zum einen ist das die blau schillernde Form ( $A$. ilia f. ilia), zum anderen die blau schillernde rötliche Form (A. ilia $\mathrm{f}$. clytie). Diesen prachtvollen Schillerglanz haben bei beiden Formen aber nur die Männchen, den Weibchen fehlt die Schillerfärbung; sie sind auf den Flügeloberseiten mit einer matten, dunkelbraunen bzw. rötlichbraunen Grundfärbung versehen. Beide Formen des Kleinen Schillerfalters haben flügeloberseits zudem eine Binde aus hellen Flecken sowie an den Außenrändern der Flügel

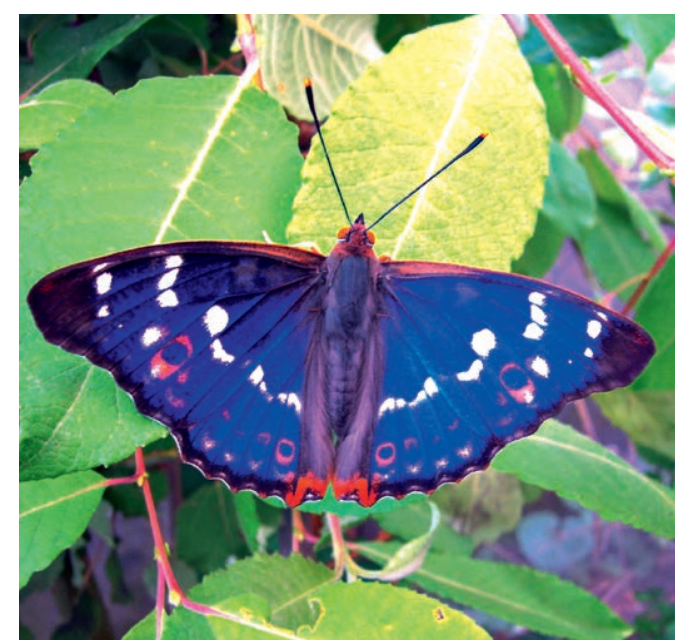

Abb. 15: Kleiner Schillerfalter (A. ilia f. ilia), Männchen. (Foto: A. Westenberger) entweder helle Punkte oder helle, schmale Bänder. Diese hellen Flecken, Punkte und Bänder sind bei f. ilia weiß und bei f. clytie ockergelb, ins rötliche gehend. Etwa in der Mitte der Vorderflügel und am Ende der Hinterflügel ist bei den Faltern außerdem ein leicht rötlich umrandeter schwarzer Augenfleck zu sehen.

Die Männchen des Kleinen Schillerfalters, die mit ihrem „Blauschiller“ auf den Flügeloberseiten von besonderer Schönheit sind, können auf Waldwegen am günstigsten bei sonnigem Wetter in den Vormittagsstunden beobachtet werden. Besonders nach vorangegangen Regennächten kann man in entsprechenden Habitaten oft mehrere Falter bei der Nahrungsaufnahme beobachten, wie sie mit ihrem gelben Saugrüssel Mineralien von feuchten Wegstellen aufnehmen. Auch tierische Exkremente auf den Wegen werden hierzu aufgesucht.

Die Weibchen sind im Gegensatz dazu nur selten auf den Waldwegen anzutreffen. Sie halten sich vor allem im Kronenbereich der Waldbäume auf und ernähren sich vorwiegend vom Saft blutender Bäume, dem Honigtau der Blattläuse, und dem Saft überreifer Früchte, wie Brombeere und Kirsche. Bei einer Zucht konnte der Mitautor dieses Artikels zudem feststellen, dass Weibchen bei der Nahrungsaufnahme auch an Regentropfen (auf Blättern) und mit Vorliebe auch

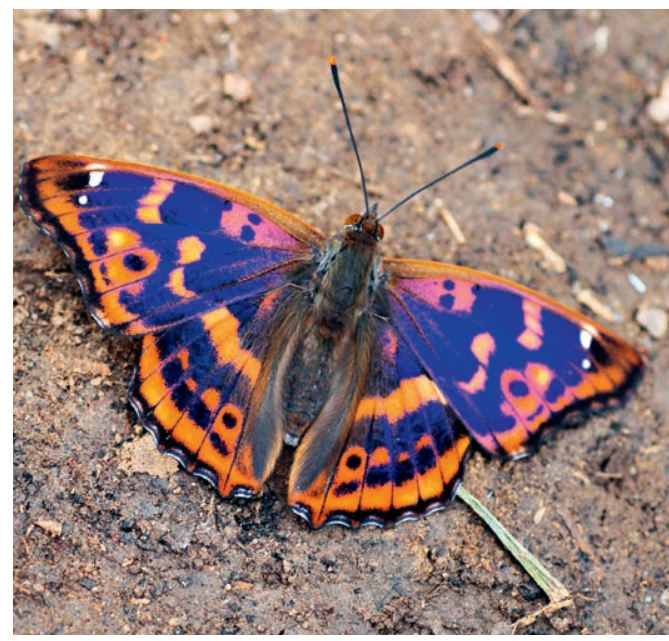

Abb. 16: Kleiner Schillerfalter (A. ilia f. clytie), Männchen. (Foto: A. Westenberger) 


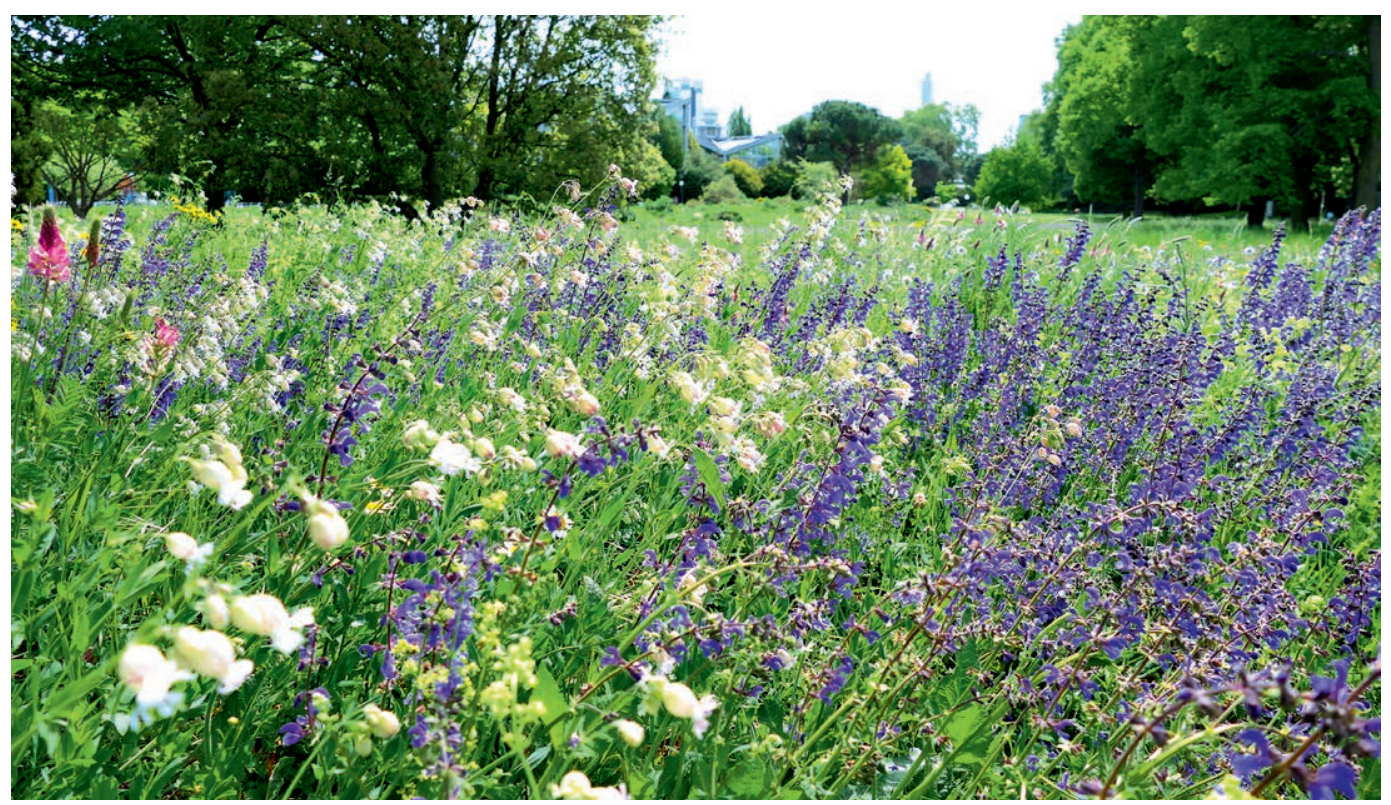

Abb. 17: Auf dieser Insektenwiese im Palmengarten sind viele Insekten, darunter auch einige Schmetterlinge, zu beobachten. (Foto: H. Steinecke)

an aufgeschnittenen Teilen einer Honigmelone saugten. Blütenpflanzen werden zur Nektaraufnahme weder von männlichen noch von weiblichen Faltern besucht. Eine Verwechslungsgefahr beim Kleinen Schillerfalter besteht nur mit dem Großen Schillerfalter, der aber größer und etwas anders gezeichnet ist. Beim Kleinen Schillerfalter überwintert die kleine Raupe (im zweiten Larvenstadium) an den Ästen von Zitter- und Schwarzpappeln.

Die Beobachtung und Förderung der Insektenvielfalt sind Bausteine des Leitthemas „Blütenbiologie“ im Palmengarten. Ein besonderes Highlight zu dieser Thematik wird im Jahr 2021 die Eröffnung des Blüten- und Schmetterlingshauses sein. In diesem wird es einen Warmhausbereich mit frei fliegenden tropischen Schmetterlingen und ihren Nahrungspflanzen sowie einen Kalthausbereich mit einer Blütenbiologie-Dauerausstellung geben.

\section{Weiterführende Literatur}

Eвеrt, G. 1991: Die Schmetterlinge Baden-Württembergs. Band 1. Tagfalter. - Stuttgart.
Mark, H. G., Peuder, W. \& Westenberger, A. 2017: Tagfalter und Widderchen der Stadt Frankfurt am Main, der Kreisgebiete Hochtaunus, Main-Taunus und der näheren Umgebung. - Königstein am Taunus, Frankfurt am Main, Hofheim-Marxheim.

Westenberger, A. \& Fabian, J. 2010: Die Tagfalter des Main-Taunus-Kreises und der näheren Umgebung, ihre Raupen und Futterpflanzen: Eine Bestandsaufnahme und Bildillustration aus den Jahren 2004-2009. - Hofheim-Marxheim.

\section{Internetseiten}

https://www.inaturalist.org/projects/tiere-und-pilze-in-frankfurts-botanischen-garten

http://www.apollo-frankfurt.de/

Entomologischer Verein Apollo

https://www.ufz.de/tagfalter-monitoring/index.php?de=41776

\section{Anschrift der Autoren und der Autorin}

Alfred Westenberger, Wilhelmstr.3, 65779 Kelkheim, E-Mail: apwestenberger@aol.com

Dr. Marco Schмidt: Palmengarten Frankfurt, Siesmayerstr. 61, 60323 Frankfurt,

E-Mail: marco.schmidt@stadt-frankfurt.de

Dr. Hilke Steinecke, Palmengarten Frankfurt, Siesmayerstr. 61, 60323 Frankfurt, E-Mail: hilke.steinecke@stadt-frankfurt.de 